\title{
Optimal Scheduling of GEO On-orbit Refuelling with Uncertain Object Satellites
}

\author{
Jing $\mathrm{Yu}^{1}$, Yong-gang $\mathrm{Yu}^{1}$, Jiang-tao Huang ${ }^{1, a}$, Xiao-qian $\mathrm{Chen}^{2}$ and Hong-yang Liu ${ }^{1}$ \\ ${ }^{1}$ China Aerodynamics Research and Development Center, Mianyang 621000, China \\ ${ }^{2}$ College of Aerospace Science and Engineering, National University of Defense Technology, \\ Changsha 410073, Hunan Province, China
}

\begin{abstract}
The mission planning of Geostationary Earth Orbit (GEO) on-orbit refuelling with uncertain object satellites is studied in this paper. Three key problems should be resolved here: Object Satellite (OSc) selection, mission sequence planning and transfer trajectory optimization. The optimization goal is to select the OSc and find the optimal servicing sequence with the best optimization objective value. Three optimization objectives, i.e. fuel cost, the total number of the OScs and the sum of the OSc priority value are taken into account. Considering this mission as a hybrid optimal control problem, a mathematical model is proposed. Multiobjective particle swarm optimization is employed to address the model. Several mission scenarios are compared and discussed. Numerical experiments indicate that 1) the method developed in this paper could address the problem efficiently; and 2) OSc distribution and varieties in optimization objective emphasis have considerable influences on the optimization results.
\end{abstract}

\section{Introduction}

Refuelling the satellites offers a great alternative for lengthening the lifetime of the constellation, as well as increasing the constellation's profitability and survivability. Furthermore, a multispacecraft rendezvous refuelling strategy can contribute significantly to improving the economic returns of onorbit service missions [1, 2].

In the past few years, many studies focused on OOR mission planning, i.e. developing optimal sequence and trajectories for SSc refuelling multiple targets. Shen [3] studies the scheduling of servicing multiple satellites in a circular orbit. Ouyang et al. [4] transformed the refuelling problem into a travel salesman problem (TSP). Zhang et al. [2, 5] focused on the LEO long-duration multispacecraft rendezvous mission. Bo et al. [6] presented a new refuelling pattern based on formation flying, and proposed two strategies to address the problem. Tsiotras and Dutta [1, 7-10] focused on OOR missions using peer-to-peer (P2P) strategy. In the investigations mentioned above, the object satellites are always definite, and much work focuses on fuel cost only. Considering the capacity limitation of the SSc, the planning problem of GEO OOR mission with uncertain OScs is studied in this paper. The OScs are undetermined before planning, and multiple relevant optimization objectives

\footnotetext{
a Corresponding author : hjtcyf@163.com
} 
will be taken into account. We consider the scheduling of GEO OOR mission as a HOC problem, and use Hybrid Automaton to model and address it.

\section{Mission scenario}

The mission studied here can be stated as follows. One SSc running on GEO belt is required to service some of the fuel-insufficient GEO satellites (see Figure 1). Since the propellant capacity of the SSc is limited, it could not service all of the satellites in a mission. The goal of the mission planning is to select the OScs that would be refuelled and find the optimal mission sequence for the SSc. In order to simplify the problem, we take two-impulse phasing maneuver and Hohmann transfer to perform orbital rendezvous, and sufficient time is given for the mission.

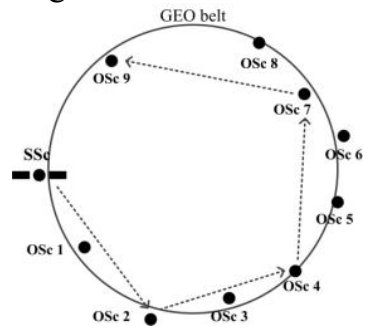

Figure 1. Mission scenario.

\section{Mathematical description of the HOC problem}

Recently, hybrid optimal control (HOC) theory has been applied to the solution of space mission planning [11-17]. The common way to model a HOC problem consists of the steps of categorical state space modelling; continuous-time dynamics modelling; continuous-valued state and control spaces modeling; discrete events modelling; and cost functions modelling. In this section, we will detail these steps.

\subsection{Categorical/Discrete state space}

Supposing there are $N$ fuel-insufficient satellites, $N_{Q}$ out of them are selected to be refuelled. Then the categorical state space for the problem is $Q=\left\{q_{1}, q_{2}, \ldots, q_{N Q}\right\}$. A directed graph (or called "digraph") can describe this space. Figure 2 shows a digraph for an example problem in which there are three possible categorical states that can be combined in some order to qualitatively describe the mission plan. The categorical state space is graphically depicted, as are the allowed transitions.

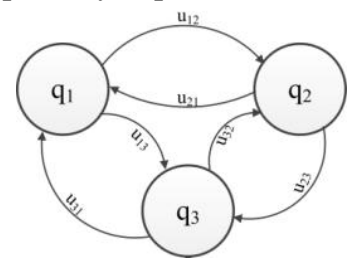

Figure 2. Digraph for the OOR mission.

In Figure 2, $N_{Q}=3$, and each vertex $q_{i}$ is the state corresponding to the SSc flying at the slot of the $i$-th OSc with zero thrust. Each edge represents a switch of the SSc's dynamics from one vector field to another. $\mathbf{u}_{\mathrm{ij}}$ denotes the system input that triggers the switch from state $q_{i}$ to $q_{j}$. A mission plan is described qualitatively by an event (or maneuver) sequence, e.g. $\mathbf{q}=\left(q_{2}, q_{1}, q_{3}\right)$, with events chosen from the categorical state space. This mission plan sequence describes that starting from the original location, the SSc refuels the OScs numbered 2, 1 and 3 one by one. The real trajectory of the SSc is $\mathbf{q}$ 
$=\left(q_{0}, q_{2}, q_{1}, q_{3}\right)$, where $q_{0}$ denotes the initial fly state of the SSc. In this paper, we tacitly approve that $q_{0}$ is the original state of the SSc, and $q_{0}$ won't appear in the mission plan.

\subsection{Continuous-time dynamics}

Associated with each state $q \in Q$ is a continuous-time controlled dynamical system: $\dot{\mathbf{x}}=f(\mathbf{x}, \mathbf{u}, t, q)$. At each discrete state, we have the dynamics of SSc with $\mathbf{x}=[\boldsymbol{r}, \boldsymbol{v}] . \boldsymbol{r}, \boldsymbol{v}$ respectively denotes the radius vector and velocity vector of the SSc. Perturbations are not taken into account [18][19] in this paper.

$$
\left\{\begin{array}{l}
\frac{d \boldsymbol{v}}{d t}=-\frac{\mu}{r^{3}} \boldsymbol{r} \\
\frac{d \boldsymbol{r}}{d t}=\boldsymbol{v}
\end{array}\right.
$$

\subsection{Continuous-valued state and control spaces}

For the problem investigated in this paper, we should consider constraints as follows.

(1) The number of satellites in the mission sequence should not be more than the total number of the fuel-insufficient satellites, i.e. $N_{Q} \leq N$.

(2) The fuel mass that the OSc receives should not be more than the mass it requires.

And (3) when the mission is completed, the remaining fuel of the SSc should be more than a defined value fuel $_{\min }$.

\subsection{Discrete events}

Let $(\mathbf{x}, \mathbf{u})$ and $\left(\mathbf{x}^{\prime}, \mathbf{u}^{\prime}\right)$ denote the continuous-valued state and control variables associated with any two vertices $q, q^{\prime} \in Q$. The generalized switching set, which may be empty, is called the event set $\mathbf{E}\left(q, q^{\prime}\right)$ and is defined (when nonempty) by means of an inequality constraint on a function $\mathbf{e}\left(\cdot, q, q^{\prime}\right)$.

$$
\mathbf{E}\left(q, q^{\prime}\right)=\left\{\left(\mathbf{x}, \mathbf{u}, \tau, \mathbf{x}^{\prime}, \mathbf{u}^{\prime}, \tau^{\prime}\right): \mathbf{e}^{L} \leq \mathbf{e}\left(\mathbf{x}, \mathbf{u}, \tau, \mathbf{x}^{\prime}, \mathbf{u}^{\prime}, \tau^{\prime}, q, q^{\prime}\right) \leq \mathbf{e}^{U}\right\}
$$

$\mathbf{e}^{L}$ and $\mathbf{e}^{U}$ are the lower and upper bounds on the values of the function $\mathbf{e}\left(\cdot, q, q^{\prime}\right)$, respectively, $\tau$ is the time corresponding to $\mathbf{x}$ and $\mathbf{u}$. The function $\mathbf{e}\left(\cdot, q, q^{\prime}\right)$ is called the event function associated with the discrete states $q$ and $q^{\prime}$.

\subsection{Cost functions}

In this paper, we consider three optimization objective functions.

(1) $J_{f}$ : fuel cost is as small as possible.

For impulsive transfers, the following expression holds between the mass of the satellite just before $\left(m\left(t_{k}^{-}\right)\right)$and just after $\left(m\left(t_{k}^{+}\right)\right)$an impulse at time $t=t_{k}$ :

$$
m\left(t_{k}^{+}\right)=m\left(t_{k}^{-}\right) e^{-\Delta v_{k} /\left(I_{s p} g_{0}\right)}
$$

Where $\Delta v_{k}$ is the gain (or loss) of the velocity due to the impulse at $t=t_{k}$.

When the SSc is running on the discrete state, there is no thrust, so the velocity increment is zero. Associated with any pair $\left(q, q^{\prime}\right) \in Q \times Q$, the $\Delta v$ consists of two components, the plane change and the in-plane transfer for the rendezvous. In this paper, we apply phasing maneuver and Hohmann transfer to in-plane transfer.

(2) $J_{N}$ : the number of satellites that will be serviced should be as large as possible.

As the SSc could not service all of the fuel-insufficient satellites, it is required that the fuelinsufficient satellites should be refueled as many as possible.

(3) $J_{p}$ : the sum of the priority value in the mission sequence should be as large as possible. 
Suppose that each fuel-insufficient satellite has a priority value. It is required that the satellite with high priority value should be serviced first.

\subsection{Summary of the HOC problem}

In summary, the On-orbit refueling mission researched in this paper could simply be described as a HOC problem, and can be formulated as:

$$
\begin{array}{ll}
\text { find } & \mathbf{q}, \mathbf{u} \\
\text { minimize } & J_{f}=\sum \operatorname{CFuel}\left(q^{i}, q^{i+1}\right) \\
\text { maxmize } & J_{N}=\operatorname{Num}(\mathbf{q}) \\
\text { maxmize } & J_{p}=\sum \operatorname{Prio}\left(q^{i}\right) \\
\text { s.t. } & \dot{\mathbf{x}}=f(\mathbf{x}, \mathbf{u}, t, q) \\
& q \in \mathbf{q}, q \in Q \\
& \operatorname{Num}(\mathbf{q}) \leq N \\
0<\text { AFuel }(q) \leq \text { RFuel }(q) & \text { Fuel }_{s}(\mathbf{q})>\text { fuel }_{\text {min }} \\
& X\left(t_{0}\right)=X_{q_{0}}
\end{array}
$$

In Eq. (4), $\mathbf{q}$ denotes the mission sequence of the SSc, and $\mathbf{u}$ gives the optimal input that could trigger the whole rendezvous trajectory with optimal cost. Equations (5)-(7) represent three optimization objectives. Equations (8) and (9) describe that each discrete state should satisfy continuous-time dynamics. Equation (10) gives the constraint that the number of satellites in the mission sequence should be not more than the total number of the fuel-insufficient satellites. Equation (11) means that the fuel mass that the OSc receives should be not more than the mass it requires, where $\operatorname{AFuel}(q)$ denotes the fuel mass the OSc receives, and $\operatorname{RFuel}(q)$ gives the fuel mass the OSc requires. Equation (12) describes that when the mission is completed, the remaining fuel of the SSc should be more than fuel $_{\text {min }}$. Equation (13) represents the initial state constraint of the SSc.

\section{Solution via particle swarm optimization}

To address the HOC model above, three key problems should be considered and resolved:

(1) OScs selection: deciding which satellite will be refuelled.

(2) Mission sequence: deciding the servicing order of the SSc.

(3) Trajectory generation: optimizing the orbital rendezvous trajectories.

Multi-Objective Particle Swarm Optimization (MOPSO) algorithm is employed to deal with the model described above. The main algorithm is provided in [15]. Here we will detail the encoding method and the fitness computation process of MOPSO.

\subsection{Encoding}

In the MOPSO algorithm used in this paper, each particle is a row matrix with $N$ columns, where $N$ denotes the total number of the fuel-insufficient satellites. Each particle contains $N$ real numbers $d_{i} \in(1,2) \quad i \in(1,2, \ldots N)$. Sorting these numbers in ascending order, the task arrangement then could be obtained. For example, if a particle is described as [1.5,1.3,1.7], it means that the SSc should service satellites 2,1 and 3 in order.

\subsection{Fitness computation}


When the particle is given, the task arrangement can be decoded, and the fitness value can be computed as follows (see Figure 3).

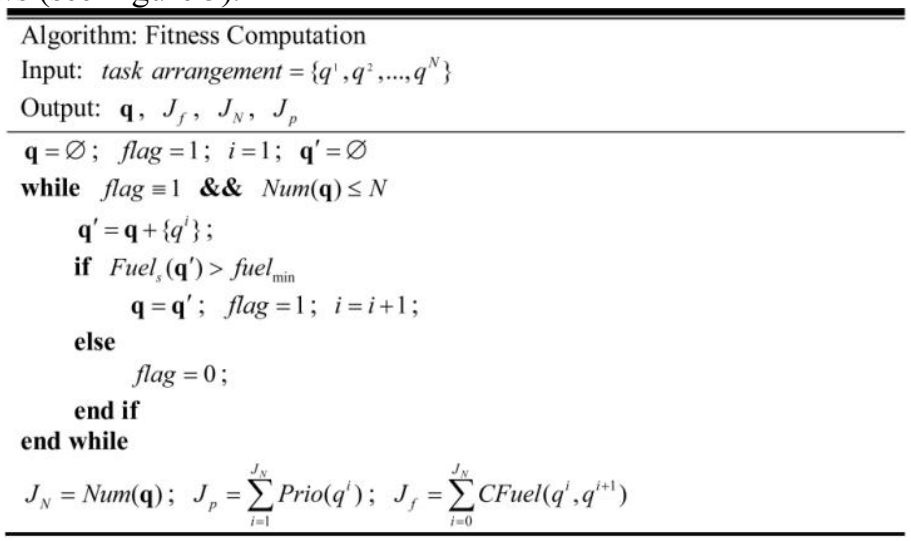

Figure 3. Fitness computation algorithm.

\section{Simulations}

\subsection{Configurations}

To demonstrate the model and the solution method, 15 objects of GEO belt were selected as fuelinsufficient satellites (see Table 1). The inclination and right ascension data are from literature [20]. Satellite dry mass, fuel requirements, and satellite priority value are given randomly.

Table 1. Parameters for GEO objects.

\begin{tabular}{ccccccc}
\hline $\begin{array}{c}\text { Satellite } \\
\text { number }\end{array}$ & name & $\begin{array}{c}\text { inclination } \\
\left({ }^{\circ}\right)\end{array}$ & $\begin{array}{c}\text { RAAN } \\
\left({ }^{\circ}\right)\end{array}$ & $\begin{array}{c}\text { True } \\
\text { anomaly } \\
\left({ }^{\circ}\right)\end{array}$ & $\begin{array}{c}\text { Fuel } \\
\text { requirement } \\
(\mathbf{k g})\end{array}$ & Priority \\
\hline 1 & Morelos 2 & 0.9128 & 90.595 & 30.171 & 600 & 1 \\
2 & Intelsat VA F15 & 1.7156 & 87.1401 & 100.814 & 600 & 4 \\
3 & TDRS 4 & 2.3935 & 82.265 & 78.521 & 700 & 6 \\
4 & Gstar 1 & 2.6347 & 80.6943 & 240.339 & 800 & 4 \\
5 & IUS-13 SRM-2 & 4.1882 & 43.4184 & 330.478 & 700 & 6 \\
6 & IUS-15 SRM-2 & 6.3096 & 70.5983 & 200.158 & 660 & 1 \\
7 & Intelsat V F5 & 7.5253 & 49.9744 & 150.229 & 720 & 4 \\
8 & Intelsat V F2 & 8.2849 & 46.1791 & 300.554 & 750 & 4 \\
9 & Intelsat 501 & 8.7881 & 44.4057 & 175.874 & 650 & 1 \\
10 & GOES 5 & 9.9086 & 39.2166 & 286.178 & 850 & 4 \\
11 & DSCS II F-16 & 10.0680 & 41.2597 & 45.231 & 600 & 6 \\
12 & IUS-2 SRM-2 & 11.2379 & 37.5653 & 123.456 & 600 & 6 \\
13 & Leasat 2 & 11.8924 & 40.1735 & 91.221 & 700 & 4 \\
14 & FLTSATCOM F4 & 12.2709 & 31.7337 & 210.369 & 800 & 1 \\
15 & DSCS II F-13 & 12.6462 & 33.5862 & 350.109 & 850 & 4 \\
\hline It is assumed that SSc and all satellites & are in circular orbits with the orbit altitude of
\end{tabular}

$r_{G}=35798 \mathrm{~km}$, and the SSc is originally located at the position where the inclination is $0 \mathrm{deg}$, the RAAN is 0 deg and the initial true anomaly is $0 \mathrm{deg}$. The initial dry mass of the SSc is $2000 \mathrm{~kg}$, while the fuel mass it initially carries is $3000 \mathrm{~kg}$. The specific impulse of the $\mathrm{SSc}$ is $300 \mathrm{~s}$, and fuel $_{\text {min }}$ here is $1 \mathrm{~kg}$. As sufficient time is provided for on-orbit refueling mission, each rendezvous could be achieved by phasing maneuver or Hohmann transfer. The time upper bound for each Phasing 
maneuver is 30 days. Four mission scenarios are given here to be compared and discussed. The SSc should return to its original location after mission in all these scenarios unless noted otherwise.

Scenario 1: all satellites and the SSc share the same orbit altitude $r_{G}$, the same inclination 0 deg and the same RAAN 0 deg.

Case 1-1: fuel demand of each satellite is $0 \mathrm{~kg}$.

Case 1-2: fuel demand of each satellite is among $[600,900] \mathrm{kg}$, as depicted in Table 1.

Case 1-3: fuel demand of satellite 3 is defined to be $1800 \mathrm{~kg}$.

Scenario 2: all satellites and the SSc share the same orbit altitude $r_{G}$, while the other parameters are as illustrated in Table 1.

Case 2-1: fuel demand of each satellite is $0 \mathrm{~kg}$; and after mission, the $\mathrm{SSc}$ is not required to return to its original location.

Case 2-2: fuel demand of each satellite is among $[600,900] \mathrm{kg}$, as depicted in Table 1.

Case 2-3: fuel demand of satellite 3 is defined to be $1800 \mathrm{~kg}$.

Scenario 3: all satellites share the same inclination 0 deg and the same RAAN 0 deg; the orbit altitude difference between two adjacent satellites is $300 \mathrm{~km}$, i.e. satellite 1 's altitude is $r_{G}$, while satellite 15 is $r_{G}-4200 \mathrm{~km}$.

Case 3-1: fuel demand of each satellite is $0 \mathrm{~kg}$; the orbit altitude of the $\mathrm{SSc}$ is initially $r_{G}$; and after mission, the SSc is not required to return to its original location.

Cade 3-2: fuel demand of each satellite is $0 \mathrm{~kg}$; the orbit altitude of the $\mathrm{SSc}$ is initially $r_{G}-4200 \mathrm{~km}$; and after mission, the SSc is not required to return to its original location.

Case 3-3: fuel demand of each satellite is depicted in Table 1; and the orbit altitude of the SSc is initially $r_{G}$.

Case 3-4: fuel demand of each satellite is depicted in Table 1; and the orbit altitude of the SSc is initially $r_{G}-4200 \mathrm{~km}$.

Scenario 4: the orbit altitude of each satellite is the same as scenario 3; and the other parameters are the same as illustrated in Table 1.

Case 4-1: fuel demand of each satellite is $0 \mathrm{~kg}$; the orbit altitude of the SSc is initially $r_{G}-4200 \mathrm{~km}$; and after mission, the SSc is not required to return to its original location.

Case 4-2: fuel demand of each satellite is depicted in Table 1; and the orbit altitude of the SSc is initially $r_{G}-4200 \mathrm{~km}$.

\subsection{Results and discussions}

When the fuel demand is $0 \mathrm{~kg}, J_{N}$ and $J_{p}$ are senseless, as all satellites can be serviced. The phase distribution of all satellites is illustrated in Figure 4. Selecting cases which take fuel demand out of account, the optimization results are shown in Table 2.

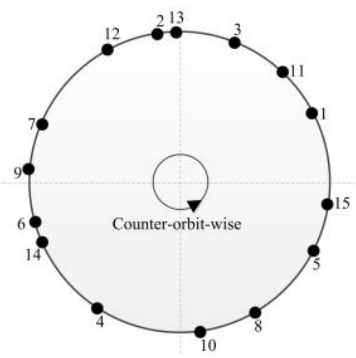

Figure 4. Phase distribution of all satellites.

Table 2. Optimization results for cases without fuel demand.

\begin{tabular}{ccc}
\hline Case & $J_{f} \mathbf{( k g )}$ & Optimal mission sequence \\
\hline $1-1$ & 146.799 & $(1,11,3,13,2,12,7,9,6,14,4,10,8,5,15)$ \\
$2-1$ & 1210.622 & $(1,2,3,4,5,6,7,9,8,10,11,12,13,15,14)$ \\
\hline
\end{tabular}




\begin{tabular}{ccc}
\hline $3-1$ & 280.234 & $(1,2,3,4,5,6,7,8,9,10,11,12,13,14,15)$ \\
$3-2$ & 272.282 & $(15,14,13,12,11,10,9,8,7,6,5,4,3,2,1)$ \\
$4-1$ & 1242.609 & $(1,2,3,4,5,6,7,8,9,10,11,12,13,14,15)$ \\
\hline
\end{tabular}

As shown in Table 2: (1) the optimization result of case 1-1 is in accordance with Shen's research [3], (2) the result of case 2-1 is in accordance with Alfriend's [20] research; (3) the results of cases 3-1 and 3-2 demonstrate that when all satellites are coplanar but not share the same altitude, the SSc always service the nearest (altitude difference is the smallest) one first; and (4) the result of case 4-1 once again verifies Alfriend's conclusion. Table 2 also verifies that the solution approach proposed in this paper can resolve the problem efficiently. Now, taking fuel demand into consideration, the nondominated frontiers for each scenario are shown in Tables 3-9.

Table 3. The non-dominated frontier for case 1-2.

\begin{tabular}{ccccc}
\hline Index & Mission sequence & $J_{f}$ & $J_{N}$ & $J_{p}$ \\
\hline 1 & $(15,5,8,10)$ & 34.726 & 12 & 4 \\
2 & $(11,3,13,2,5)$ & 66.773 & 26 & 5 \\
3 & $(11,3,13,2,12)$ & 61.597 & 24 & 5 \\
4 & $(15,5,11,3,13)$ & 57.032 & 23 & 5 \\
5 & $(15,1,11,3,13)$ & 47.559 & 18 & 5 \\
6 & $(1,11,3,13,2)$ & 49.960 & 21 & 5 \\
\hline
\end{tabular}

Table 4. The non-dominated frontier for case 1-3.

\begin{tabular}{ccccc}
\hline Index & Mission sequence & $J_{f}$ & $J_{N}$ & $J_{p}$ \\
\hline 1 & $(1,3,13)$ & 47.69 & 13 & 3 \\
2 & $(1,11,13,3)$ & 48.43 & 17 & 4 \\
3 & $(1,11,13,2,3)$ & 52.17 & 21 & 5 \\
4 & $(5,11,13,2,3)$ & 72.18 & 26 & 5 \\
5 & $(15,5,8,10)$ & 34.73 & 12 & 4 \\
6 & $(15,5,11,13,3)$ & 57.93 & 23 & 5 \\
7 & $(11,13,2,12,3)$ & 65.97 & 24 & 5 \\
8 & $(15,5,1,11,3)$ & 50.29 & 18 & 5 \\
9 & $(15,5,8,1,11)$ & 49.19 & 16 & 5 \\
\hline
\end{tabular}

Table 5. The non-dominated frontier for case 2-2.

\begin{tabular}{ccccc}
\hline Index & Mission sequence & $J_{f}$ & $J_{N}$ & $J_{p}$ \\
\hline 1 & $(5,8,7,3)$ & 606.37 & 20 & 4 \\
2 & $(5,8,7,2)$ & 605.62 & 18 & 4 \\
3 & $(1,2,3,4)$ & 255.68 & 15 & 4 \\
4 & $(1,2,3,5,4)$ & 371.01 & 21 & 5 \\
5 & $(2,3,5,13)$ & 717.20 & 22 & 4 \\
\hline
\end{tabular}

Table 6. The non-dominated frontier for case 2-3.

\begin{tabular}{ccccc}
\hline Index & Mission sequence & $J_{f}$ & $J_{N}$ & $J_{p}$ \\
\hline 1 & $(2,4,5,3)$ & 449.41 & 20 & 4 \\
2 & $(5,8,13,3)$ & 761.81 & 22 & 4 \\
3 & $(1,2,5,3)$ & 401.75 & 17 & 4 \\
4 & $(2,3,5)$ & 314.07 & 16 & 3 \\
5 & $(1,3,2)$ & 195.61 & 11 & 3 \\
6 & $(1,2,4,3)$ & 282.68 & 15 & 4 \\
7 & $(2,3,4)$ & 258.76 & 14 & 3 \\
\hline
\end{tabular}

Table 7. The non-dominated frontier for case 3-3. 


\begin{tabular}{ccccc}
\hline Index & Mission sequence & $J_{f}$ & $J_{N}$ & $J_{p}$ \\
\hline 1 & $(2,3,5,7,13)$ & 232.61 & 26 & 5 \\
2 & $(1,2,3,4,5)$ & 92.41 & 21 & 5 \\
3 & $(2,3,4,1)$ & 85.20 & 15 & 4 \\
4 & $(2,3,4,5,7)$ & 121.09 & 24 & 5 \\
\hline
\end{tabular}

Table 8. The non-dominated frontier for case 3-4.

\begin{tabular}{ccccc}
\hline Index & Mission sequence & $J_{f}$ & $J_{N}$ & $J_{p}$ \\
\hline 1 & $(14,13,12,11,5)$ & 198.96 & 24 & 5 \\
2 & $(15,14,12,11,13)$ & 94.37 & 19 & 5 \\
3 & $(14,13,12,11,8)$ & 149.45 & 22 & 5 \\
4 & $(15,14,13,12)$ & 71.66 & 15 & 4 \\
5 & $(13,12,11,5,3)$ & 259.92 & 26 & 5 \\
\hline
\end{tabular}

Table 9. The non-dominated frontier for case 4-2.

\begin{tabular}{ccccc}
\hline Index & Mission sequence & $J_{f}$ & $J_{N}$ & $J_{p}$ \\
\hline 1 & $(4,3,2,1)$ & 546.21 & 15 & 4 \\
2 & $(2,3,4,5)$ & 622.16 & 20 & 4 \\
3 & $(2,3,5,13)$ & 979.64 & 22 & 4 \\
\hline
\end{tabular}

Conclusions can be obtained from Table 3-Table 9 that:

(1) When priority is given to optimization objective $J_{p}$, satellite which has high priority value would be serviced first. Taking 1-2(2), 1-3(4), 2-2(5), 2-3(2), 3-3(1), 3-4(5) and 4-2(2) as examples, if it is possible, the SSc always service satellite 3, 5, and 13 first.

(2) When priority is given to optimization objective $J_{N}$, satellite which demands small fuel mass and consumes small in orbital transferring would be serviced first. Taking 1-2(4) and 1-3(6) as examples, in order to fuel one more satellite, the SSc services satellite 12, 5, 11, 13 first, and then satellite 3. Since the fuel demand of satellite 3 is large, if it is serviced first, there would not be sufficient fuel to satisfy other 4 satellites.

(3) When priority is given to optimization objective $J_{f}$, in most cases, satellite that demands large fuel mass would be serviced first. That is due to the fact that the smaller the $J_{N}$ is, the less the orbital transfer would consume (see 1-1(1), 2-3(5) and 2-3(7)).

(4) When orbital plane differences are considered, the optimal servicing order is still mainly determined by orbital-plane differences (see tables 7, 8, and 9).

\section{Conclusions}

Considering the practical fact that the SSc capacity is limited and not all of the fuel-insufficient satellites could be refuelled in a mission, the problem of optimal scheduling of GEO OOR mission with uncertain OScs is studied. Specifically, the SSc is considered to be initially on the GEO belt. Numerous fuel-insufficient satellites request OOR at the same time. The SSc is unable to service all of these satellites. It just rendezvouses with some selected ones and refuels them. Phasing maneuver and Hohmann transfer are employed for each rendezvous. The optimization goal is to select the servicing targets and find the optimal servicing sequence with the best optimization objective value. Three optimization objectives, i.e. fuel cost, the total number of the servicing targets and the sum of the OSc priority value are taken into account. Considering this mission as a hybrid optimal control problem, a mathematical model is proposed. MOPSO is employed to address the model. Several OOR scenarios are compared in this paper. Numerical experiments indicate that 1) the method developed in this paper could address the problem efficiently; and 2) varieties in optimization objective emphasis and satellite distribution have considerable influences on the optimization results, and these influences appear regularly. 


\section{Acknowledgment}

This work was supported by National Natural Science Foundation of China under Grant No. 71601179 .

\section{References}

1. Tsiotras, P., and Nailly, A., Comparison Between Peer-to-Peer and Single Spacecraft Refueling Strategies for Spacecraft in Circular Orbits, Infotech at Aerospace Conference, AIAA Paper 2005-7115,Arlington, VA, Sept. 2005

2. J. Zhang, G.T. Parks, Y.Z. Luo, et al. Multispacecraft Refueling Optimization Considering the J2 Perturbation and Window Constraints. Journal of Guidance, Control, and Dynamics. 37(1) (2014): 111-122.

3. H. Shen, P. Tsiotras, Optimal Scheduling for Servicing Multiple Satellites in a Circular Constellation, in: AIAA/AAS Astrodynamics Specialists Conference and Exhibit, (Monterey, CA), Aug. 2002.

4. Q. Ouyang, W. Yao, X.Q. Chen, Mission Programming of On-orbit Re-fueling for Geosynchronous Satellites (in Chinese), J. Astronaut 31 (2010) 2629-2634.

5. J. Zhang, Y.Z. Luo, G.J. Tang, Hybrid planning for LEO long-duration multi-spacecraft rendezvous mission, Sci. China Tech. Sci. 55 (2012) 233-243.

6. X. Bo, Q. Feng, Research on constellation refueling based on formation flying, Acta Astronautica 68 (2011) 1987-1995.

7. Dutta A, Arora N, Russell RP. Peer-to-Peer Refueling Strategy Using Low-Thrust Propulsion [J]. Journal of Spacecraft and Rockets, 2012, 49(5): 944-954.

8. Shen, H., and Tsiotras, P.,"Peer-to-Peer Refueling for Circular Satellite Constellations,"Journal of Guidance, Control, and Dynamics, Vol. 28, No. 6, 2005, pp. 1220-1230. doi:10.2514/1.9570

9. Dutta, A., and Tsiotras, P.,"Egalitarian Peer-to-Peer Satellite Refueling Strategy,"Journal of Spacecraft and Rockets, Vol. 45, No. 3, 2008, pp. 608-618. doi:10.2514/1.31299

10. Dutta, A., and Tsiotras, P.,"Network Flow Formulation for Cooperative Peer-to-Peer Refueling Strategies,"Journal of Guidance, Control, and Dynamics, Vol. 33, No. 5, 2010, pp. 1539-1549

11. B.A. Conway, C.M. Chilan, B.J. Wall, Evolutionary Principles Applied to Mission Planning Problems, Celestial Mechanics and Dynamical Astronomy 97(2) (2007): 73-86.

12. I.M. Ross, C.N. D'Souza, Hybrid Optimal Control Framework for Mission Planning, Journal of Guidance, Control, and Dynamics 28(4) (2005): 686-697.

13. C.M. Chilan, Automated Design of Multiphase Space Missions Using Hybrid Optimal Control. Ph.D. diss., University of Illinois at Urbana-Champaign, 2009.

14. B.J. Wall, B.A. Conway, Genetic Algorithms Applied to the Solution of Hybrid Optimal Control Problems in Astrodynamics, J. Glob. Optim. 44 (2009): 493-508.

15. Jing Y, Chen $\mathrm{X}$, Chen L. Biobjective planning of GEO debris removal mission with multiple servicing spacecrafts [J]. Acta Astronautica, 2014, 105(1): 311-320.

16. Jing Yu, Xiao-qian Chen, Li-hu Chen, Dong Hao. Optimal scheduling of GEO debris removing based on hybrid optimal control theory [J]. Acta Astronautica, 2014, 93: 400-409.

17. Jing Yu, Xiao-qian Chen, Li-hu Chen. Optimal planning of LEO active debris removal based on hybrid optimal control theory [J]. Advances in Space Research, 2015, 55(11): 2628-2640.

18. H. L. Shi, Y. B. Han, L. H. Ma, et al., Beyong Life-Cycle Utilization of Geostationary Communication Satellites in End-of-Life, Satellite Communications (2010) 323-365.

19. L. J. Friesen, A. A. Jackson, H. A. Zook, et al., Analysis of Orbital Perturbations Acting on Objects in Orbits Near Geosynchronous Earth Orbit, Journal of Geophysical Research 97 (1992) 3845-3863.

20. Alfriend KT, Lee D, Creamer NG. Optimal Servicing of Geosynchronous Satellites. Journal of Guidance, Control, and Dynamics, 2006, 29(1): 203-206. 